\title{
Filosofía de la danza: Cuerpo y expresión simbólica
}

\author{
Philosophy of Dance: Body and Symbolic Expression
}

\author{
ANÍBAL MONASTERIO ASTOBIZA*
}

\begin{abstract}
Resumen: La filosofía de la danza se define como el estudio sobre un cierto tipo de arte no-representativo que a través del movimiento del cuerpo alcanza efectos estéticos. Desde la aproximación de las ciencias cognitivas se desmiente esta presuposición original de la danza como arte norepresentativo. Se introducen, brevemente, varias razones para ver la filosofía de la danza desde el enfoque naturalista de las ciencias cognitivas donde el cuerpo tiene capacidad de expresión simbólica.
\end{abstract}

Palabras claves: cuerpo, filosofía de la danza, ciencias cognitivas.

\begin{abstract}
Philosophy of dance is defined as the study of a sort of non-representational art which through body movements elicits some aesthetics responses. From a cognitive science approach this assumption is doubtful. In this paper is briefly introduced several reasons to view the philosophy of dance from the perspective of the naturalistic approach of cognitive science where the body is described as capable of symbolic expression.
\end{abstract}

Keywords: body, philosophy of dance, cognitive science.

\section{El yo corporal y la filosofía de la danza}

El cuerpo es un concepto anatómico de la medicina, una estructura física y un medio de codificación semiótico. Pero además, el desarrollo de nuestra identidad personal, de nuestro sentido del yo (ipseidad), está íntimamente relacionado con nuestro cuerpo. Nuestro cuerpo es sumamente importante. Es a través de él como conocemos la realidad física y a otras

Fecha de recepción: 14/06/2016. Fecha de aceptación: 27/07/2016.

* Investigador colaborador IFS-CSIC. Trabajo realizado en el marco de los proyectos de investigación KONTUZ!: Responsabilidad causal de la comisión por omisión: Una dilucidación ético-jurídica de los problemas de la inacción indebida (MINECOFFI2014-53926-R); y el proyecto de investigación: La constitución del sujeto en la interacción social: identidad, normas y sentido de la acción desde la perspectiva de la filosofía de la acción, la epistemología y la filosofía experimental (FFI2015-67569-C2-2-P) Las líneas de investigación del autor son: Filosofía de la Ciencia Cognitiva, Cognición social, Bioética/Neuroética (tecnologías de biomejora), Ética Experimental, Ética robótica y del Big Data, Psicología Moral, Filosofía de la Neurociencia, Filosofía de la Psiquiatría,Teoría de la Acción/agencia, comportamiento animal colectivo, Cooperación y Altruismo. Autor de los artículos: Monasterio Astobiza, A., y Ezquerro Martínez, J. (2013). El sistema de neuronas espejo y el procesamiento facial de las emociones: El caso del miedo.Ciencia Cognitiva 7:2, 34-36 y Monasterio Astobiza A. (2016), La cognición social de las alucinaciones auditivas verbales Quaderns de Psicología Vol. 18, No 1 , 63-73. E-mail: anibalmastobiza@gmail.com 
personas. Conocemos la realidad física y material de los objetos, porque a través de nuestro cuerpo interactuamos con ellos. Conocemos a otras personas, no solo porque tienen mente y les atribuimos estados mentales, sino porque poseen cuerpo: un cuerpo como el nuestro.

Una de las características principales de nuestro cuerpo es que se mueve espaciotemporalmente. Nuestro cuerpo es un objeto más entre otros objetos y al mismo tiempo una experiencia sentida. El movimiento del cuerpo de otros es percibido, pero nuestro propio movimiento es sentido. Aunque también nuestro propio cuerpo lo vemos.

El cuerpo se usa, se representa y se es consciente de él. Como se verá más adelante la danza es la práctica y ejercicio físico que intensifica la relación entre estos tres elementos en un bucle interactivo a través de la atención como proceso psicológico básico. Estas funcionalidades según algunos autores varían en grado de persona a persona. La consciencia de nuestro cuerpo, nuestra agencia, no se desarrolla aislada de otras estructuras y procesos cognitivos: el conocimiento de los objetos físicos, de los cuerpos humanos y los estados psicológicos están interrelacionados (Meltzoff 2013, 139).

$\mathrm{Al}$ ver y sentir nuestro propio cuerpo y ver, pero no sentir, el cuerpo de los demás somos capaces de establecer una correspondencia de que el cuerpo que vemos es como el nuestro $\mathrm{y}$, por consiguiente, entender que lo que sentimos nosotros también lo sienten los otros. Gracias al proceso cíclico acción-percepción y en concreto a la actividad de un grupo especial de neuronas llamadas neuronas espejo (de ahora en adelante NE) por su capacidad de activarse simultáneamente cuando se ve una acción dirigida hacia un objeto físico y cuando el individuo ejecuta la misma acción (Gallese V., Fadiga L., Fogassi L. y Rizzolatti G.. 1996, 593), (Rizzolatti G., Fadiga L. Gallesse V. y Fogassi L.1996a, 131), (Rizzolatti 2004, 169); podemos incluso entender las intenciones de las acciones y ser capaces de imitarlas.

Los movimientos del cuerpo de los demás son significativos para nosotros en términos de nuestra capacidad para poder realizarlos. Gracias a las NE la distancia entre el yo y el tú se cubre y como resultado de ser la base neurofisiológica de entender la intencionalidad de las acciones podemos reconocer las emociones, el dolor y ser empáticos. Para la danza lo más importante de las funciones de estas estructuras neuronales es que permiten la sincronización y coordinación con otra persona durante el baile.

Dentro de la filosofía de la ciencia cognitiva a toda la serie de propuestas que consideran al cuerpo como una parte principal a la hora de entender la cognición se les llama "cognición encarnada" o "cognición situada". Algunas de sus afirmaciones actualmente pueden ser consideradas indeterminadas o no verificables, como la tesis de la mente extendida (Clark y Chalmers 1998,7$)$ que afirma que cualquier medio extracortical o extracraneal con ciertas características ayuda, potencia y hasta, a veces, suplanta nuestra cognición; pero en términos generales la posición es correcta. Sin el cuerpo, no se puede entender la cognición y viceversa.

La danza es la práctica y arte del cuerpo en movimiento siguiendo, normalmente, el ritmo de la música. La filosofía de la danza es el área de estudio de la práctica artística de la danza que desde un punto de vista naturalista estudia las bases y evidencias científicas que disponemos sobre la percepción del cuerpo, memoria corporal, control motor, percepción multisensorial de la música y el movimiento etc.

La filosofía de la danza también se centra en los aspectos estéticos y huelga decir que la filosofía de la danza está poco representada en la estética contemporánea por la falta de metodologías y marco teórico armonizado con el de otros objetos de estudio tradicionales de 
la estética como la pintura, música etc. La filosofía de la danza con un enfoque naturalista también comparte intereses con la ciencia cognitiva de la danza que investiga científicamente el movimiento del cuerpo humano, así como los procesos cognitivos que lo permiten.

Fíjense que se dice filosofía de la danza desde una aproximación naturalista o ciencia cognitiva de la danza porque se parte de la idea de que el arte, y en concreto la danza, se puede explicar desde dos puntos de vista diferentes. Existe una explicación cultural de la danza por la cual todo es cultural que contrasta con una aproximación bio-cultural que dice que es la naturaleza humana y por tanto incluye factores biológicos. Desde el enfoque biocultural o naturalista, la danza es el producto de procesos cognitivos, motores, perceptuales y afectivos seleccionados por la evolución.

En este sentido, desde una aproximación naturalista en la filosofía de la danza y la experiencia artística de la danza, siguiendo a Noel Carroll $(2004,61)$ y su descripción general de las características del arte, podemos identificar una serie de atributos sui generis de la danza y otros que pueden ser compartidos con otras formas de arte y su experiencia estética:

- la danza se reconoce transculturalmente: aparece independientemente en distintas culturas y épocas

- la danza coordina los sentimientos de las personas: adapta a la audiencia unos con otro-promueve la cohesión del grupo aumenta la sensibilidad hacia los sentimientos de los demás

- el arte, incluida la danza, ayuda a construir una identidad social

- el arte, incluida la danza, entrena la cognición social y ayuda a mejorar nuestra capacidad para "leer la mente" de los demás detectando las intenciones y emociones de otros

Pero a diferencia de otras formas de arte donde procesos cognitivos de toma de perspectiva, emociones ficticias o empatía nos relacionan con las obras de arte de una forma indirecta, aunque cada vez más se está incorporando al cuerpo: mecanismos de simulación de acciones y sensaciones corpóreas en la apreciación del arte (Gallese y Cuccio 2015); solo en la danza gracias a la coordinación y sincronización del movimiento emerge una experiencia mutua y una intersubjetividad confirmada por los gestos, emociones y expresiones del compañero o compañera de baile y la observación de estos por parte del espectador.

\section{Las diferentes instanciaciones del cuerpo}

El cuerpo no solo es una estructura física y material, recibe múltiples consideraciones sociales. Al ser parte de nuestra identidad personal lo utilizamos como un medio de comunicación o vehículo semiótico. Esta faceta se despliega plenamente en la danza. Bailamos con propósitos educativos y pedagógicos, sociales, militares, y hasta deportivos.

Las instanciaciones del cuerpo en la danza son múltiples, pero se puede apuntar algunas. Una lista no exhaustiva, pero si comprehensiva, es esta de Bresnahan (2015):

1. Danza como una de las bellas artes (pace Hegel)

2. Danza no limitada a un proscenio, sino también en una galería de arte, danza cómica, danza en la calle 
3. Danza como terapia (por ejemplo para fortalecer la musculatura y prepararse para el parto)

4. Danza como ejercicio físico (en los gimnasios etc.)

5. Danza social en los bares, bodas...

6. Danza como forma de preservar la tradición cultural (danza china)

7. Danza competitiva (break dance)

8. Danza de guerra (Haka Maori, africana etc.)

9. Danza improvisada (que transversaliza a todas)

De acuerdo con Bresnahan (2015) una definición filosófica estricta de la danza es complicada. Para filósofos pragmatistas, procesuales o fenomenólogos una definición del tipo para qué sirve y cuáles son las funciones de la danza no serviría de la misma forma que para un filósofo analítico. Bresnahan ha hablado en alguna ocasión de la esencia inefable de la danza. Es legítimo defender esta posición, pero creo que es preferible ir describiendo los elementos que componen el fenómeno de la danza y, si es posible, ofrecer una definición generalizable de ella.

Le debemos a Kant la noción de trascendentalidad como el estudio de las condiciones de posibilidad de los fenómenos. Un enfoque filosófico o científico cognitivo no se diferencia cuando trata cuestiones trascendentales sobre la danza, es decir, qué hace que algo sea considerado danza y no otra cosa. A partir de ahí, se puede construir una teoría y lanzar predicciones que se podrán falsear.

En principio, la definición de danza como un arte no-representativo no casa con los datos de la ciencia cognitiva que interseccionan con la danza. Veamos porqué.

\section{Cuerpo como expresión simbólica}

Como reconoce el investigador Ivar Hagendoorn $(2011,523)$ la danza es un universal cultural humano y citando a Mallarmé plantea la cuestión simbólica de la danza:

"cómo un bailarín escribiendo con su cuerpo, sugiere cosas que la palabra escrita solo puede expresar en varios párrafos de diálogo o prosa descriptiva".

La tradicional definición de la danza como un arte no-representativo que a través del movimiento del cuerpo alcanza efectos estéticos, es correcta solo a medias. Porque la danza es un arte representativo y simbólico. La danza nos habla.

El sistema de NE que como se ha dicho más arriba es una clase especial de neuronas que se activan tanto cuando se observa la realización de una acción por parte de otro individuo como cuando se ejecuta esa misma acción, originariamente descubiertas en el área F5 de la corteza motora del cerebro del macaco; se cree gracias a diversas evidencias que también se encuentran en el cerebro humano.

Estructuras corticales que comprenden el sistema de NE son el pars opercularis del giro inferior frontal y el lóbulo parietal inferior rostral que se activan con la observación/ ejecución de:

- acciones dirigidas a objetos 
- acciones comunicativas

- movimientos corporales

También se han encontrado NE es estructuras subcorticales como la ínsula, amígdala y área somatosensorial primaria y secundaria, que se activan durante la experiencia/observación de:

- emociones

- sensaciones

Con esta doble funcionalidad de las NE, base neurofisiológica del entendimiento de la experiencia agencial, los movimientos del cuerpo en la danza dejan de ser simples movimientos para convertirse en acciones y aún más, en expresiones simbólicas que, por tanto, tienen significado.

La danza no tendría sentido si los movimientos corporales no fueran inteligibles y produjeran ciertas emociones a través del movimiento del cuerpo, incluso hasta en la danza más improvisacional y desestructurada contemporánea. Porque los bailarines y bailarinas tienen un cuerpo similar al mío yo entiendo sus movimientos en la medida que también tengo la capacidad de realizarlos y sentir lo que se siente cuando actúo. Bien es cierto, que a mayor repertorio comportamental y habilidad en el control del movimiento, mejor será el entendimiento de las acciones (Calvo-Merino B., Glaser D., Grezes J., Passingham R. y Haggard P. 2005 , 1245). Estos es así porque la imitación, reproducir lo que veo, es un mecanismo que permite entender, hacer mío y por tanto dotar de sentido, las acciones de otros individuos.

El campo de la neuroestética (Zeki 2009, Kandel 2016) que viene a ser el estudio de las bases neuronales de la apreciación y juicio estético se ha interesado inicialmente en el arte visual, pero poco a poco se ha ido interesando en otras formas y géneros de arte como la danza. La danza es una forma de arte muy interesante para explorar y no solo es estudiado desde la neuroestética de la danza, sino desde un cuerpo de conocimientos amplio que investiga cómo la experiencia de las acciones modula la percepción.

La neuroestética de la danza está idealmente posicionada para explorar la idea de que es a través del cuerpo con mecanismos de simulación de acciones y sensaciones corpóreas como apreciamos el arte y, particularmente, la danza. Desde este enfoque corporizado o encarnado la activación de un sistema de observación de acciones (que comprenden estructuras occipitotemporales, parietales y frontales encontrándose también las NE) es fundamental en la respuesta estética.

Un aspecto crítico de esta hipótesis de la percepción encarnada o corporizada del arte y particularmente de la danza es la idea de que la simulación de las acciones, sensaciones corporales y emociones visibles o implicadas en la obra de arte (un ballet, baile o movimiento rítmico del cuerpo) influye en la experiencia estética del observador.

Freedberg y Gallese $(2007,198)$ sugieren que la resonancia corporal del arte en el observador está causada por el contenido de la obra de arte y ponen como ejemplo el cuadro de Goya "Qúe hay que hacer más" donde se ve un cuerpo torturado que produce en el espectador una empatía cinestésica de dolor. 


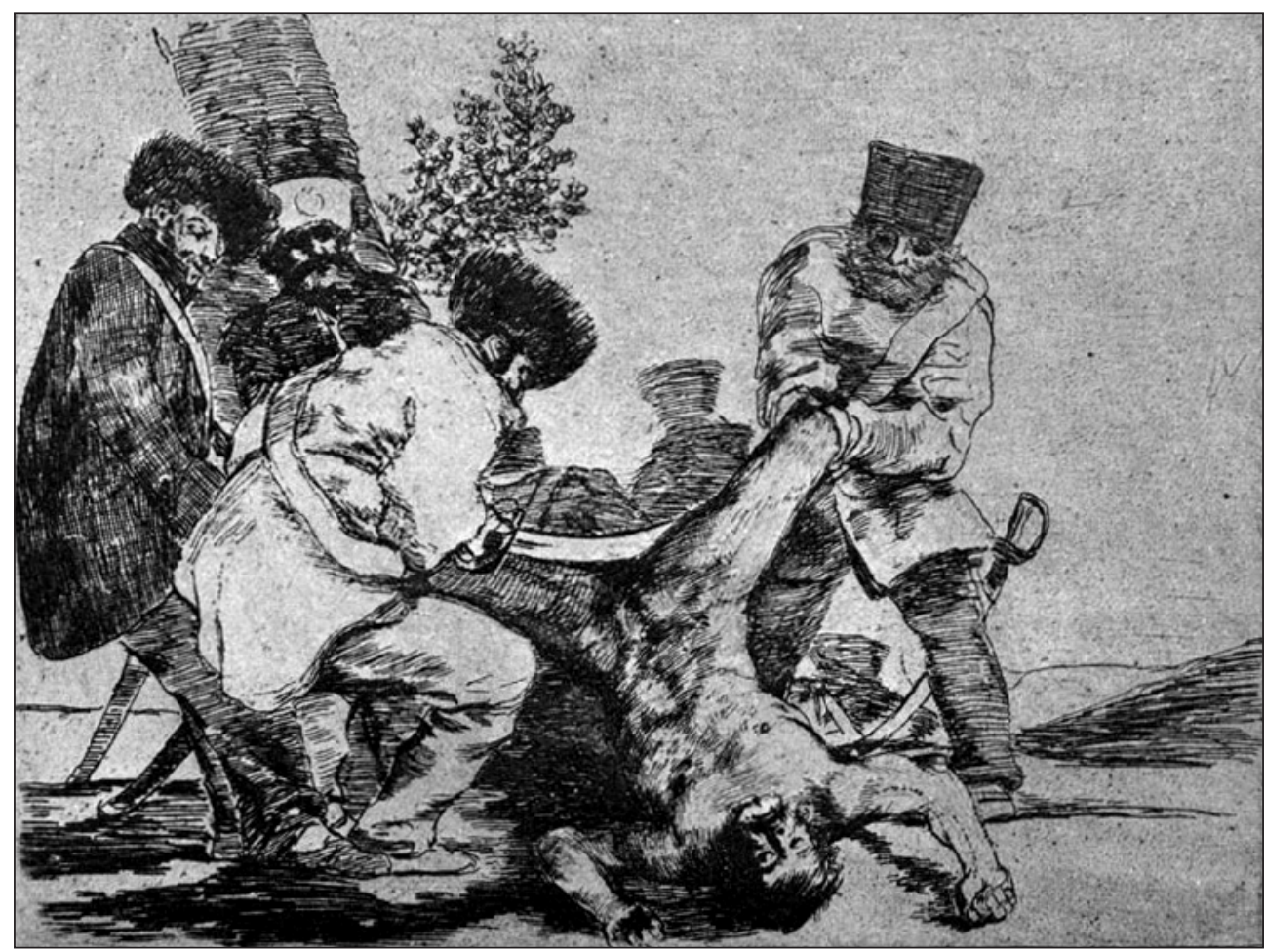

Desastres de la Guerra 33 ¿Qué hay que hacer más?, 1810-184. Francisco José de Goya y Lucientes

Freddberg y Gallese se preguntan si la simulación encarnada o corporizada de la experiencia estética de la obra de arte difiere de nuestras respuestas empáticas ante las acciones de la vida real. Es decir, qué diferencia existe en nuestra simulación corporizada cuando vemos acciones representadas en cuadros o esculturas de acciones reales.

La danza, como forma de arte, es ideal para poder contestar a esta y otras cuestiones sobre la perspectiva encarnada o corporizada en la neuroestética. Hasta la fecha existen numerosos estudios que afirman la superposición de la percepción de las acciones y la ejecución de las mismas. El sistema de NE es un ejemplo de ello. Se ha demostrado la activación corticoespinal del observador de movimientos y acciones de otro, medido a través de potenciales evocados motores que son las respuestas neurofisiológicas del sistema sensoriomotor ante un estímulo específico (Fadiga et al. 1995).

También se ha estudiado cómo la experiencia física o visual previa del observador influye en la percepción de las acciones de otros. Un hallazgo consistente y robusto dentro de esta línea de investigación es que cuando bailarines expertos observan movimientos de otros bailarines que ellos mismos son buenos, a la hora de realizarlos hay una fuerte activación en el sistema cerebral encargado de representar las acciones (Calvo-Merino et al. 2005, 1245).

El cuerpo se comunica gestualmente y la danza es una expresión simbólica. Es interesante el proyecto de Christiensen, Nadal, Cela-Conde y Gomila $(2014,181)$ de a partir 
de la forma histórica de los movimientos de la danza artística y el sistema de notación del movimiento por parte del coreógrafo de origen húngaro Rudolf Laban, crear una batería de estímulos normativa para investigar cómo el cerebro procesa el movimiento, cómo se perciben las emociones a través del cuerpo y cómo la danza puede ser una fuente de experiencia estética.

Futuros investigadores que hagan uso de esta batería de estímulos ecológicamente válidos de 203 movimientos de baile, no harán más que constatar que el cuerpo tiene poder de expresión simbólica.

\section{Danza, cuerpo y simbolismo: Hacia una nueva filosofía de la danza}

¿Qué entendemos por simbólica cuando decimos "expresión corporal simbólica" de la danza? De acuerdo con el filósofo Charles S. Pierce hay tres tipos de signos: índices, iconos y símbolos. Todo signo es un estímulo con significado. La diferencia se encuentra en el modo en que los signos se asocian con el significado o mejor dicho con el objeto al que se refieren.

Un índice (señalar con el dedo) es el signo que directamente apunta a algo que significa porque se correlaciona con él. Un icono (una pintura figurativa o fotografía) físicamente se asimila a lo que representa y finalmente un símbolo (una palabra) es todo signo que activa una red de asociaciones de otros conceptos y habitualmente adquiere su significado por convención.

Esta clásica taxonomía, un tanto simplificada pero pertinente para nuestros propósitos, de los signos viene a ser básica en la teoría semiótica y de la comunicación. Si la extrapolamos a los movimientos corporales de la danza cuando decimos que las acciones del bailarín o bailarina son simbólicas, estamos simplemente diciendo que representan, que tienen significado. Pero a diferencia de la noción tradicional de símbolo, no es un significado arbitrario. Hay un fenómeno estudiado por la neurociencia cognitiva que se llama "movimiento biológico" que es más apropiado para definir el tipo de significado que ofrecen los movimientos corporales en la danza.

El movimiento biológico es un fenómeno de nuestro sistema visual que permite obtener y reconocer información dispersa de un objeto, en este caso del cuerpo biológico humano o no-humano. Por "movimiento biológico" podemos entender la animación o movimiento autopropulsado que tienen las criaturas vivas. Johansson $(1973,202)$ colocó estratégicamente puntos de luz en las articulaciones del cuerpo de una persona. Con estas luces que crean una silueta degradada de la apariencia virtual de un cuerpo se puede discriminar la agencia del cuerpo. Cada una de las luces por separado no aporta información alguna, se mueven translacional o elípticamente. Pero todas en su conjunto generan información de movimiento espacio-temporal a partir del cual los observadores pueden identificar el género del actor (Kozlowski y Cutting 1977, 576), la actividad que realiza (Johansson 1973), e incluso el estado emocional (Brownlow S., Dixon A., Egbert C. y Radcliffe R. 1997, 411).

Este movimiento biológico que nuestro sistema visual está preparado para obtener es el que crea la fuerza de la expresión simbólica de los movimientos y acciones corporales en la danza. En otras palabras, su sentido y significado.

Por el mero hecho de percibir el cuerpo en movimiento, el fenómeno del movimiento biológico sumado al sistema de NE y otras áreas que permiten simular en nuestra propia 
mente las acciones de los demás al observarlas, ya permiten de una forma inmediata dotar de sentido (e intencionalidad y agencia) y por tanto expresión simbólica, a la danza.

El enfoque naturalista de la ciencia cognitiva de la danza presenta suficientes evidencias empíricas traídas de diferentes áreas de estudio para mostrar que la danza es una forma de arte representativo que produce efectos estéticos porque el cuerpo tiene expresión simbólica. Esta es la primera tesis que permite caminar hacia una nueva filosofía de la danza:

- el cuerpo se expresa simbólicamente.

Decir que el cuerpo se expresa simbólicamente es decir que el cuerpo y sus movimientos tienen un significado fácilmente interpretable por el observador. El movimiento es intencional.

En la danza el movimiento corporal se convierte en un superestímulo o estímulo supernormal una idea del etólogo Niko Tinberger, premio Nobel de fisiología o medicina y, por cierto, perteneciente a la única familia que tiene dos premios nobeles en sus filas ya que su hermano Jan Tinberger recibió el Nobel de economía junto a Ragnar Frish en 1969, que hace que su interpretación simbólica sea directa y fácil. Un superestímulo es una versión exagerada de un estímulo al cual hay una tendencia a responder. Tinberger estudió como algunas especies de aves sus hembras son capaces de incubar huevos falsos porque un rasgo de los huevos naturales es amplificado en los falsos, por ejemplo, el color. Estas hembras dejaban sus huevos para incubar los falsos incluso aunque su tamaño fuera el doble de grande y resbalaran por él. Con otras especies de peces pasaba exactamente lo mismo. Los peces eran engañados por peces de madera atacándolos a estos cuando se acercaban en lugar de a otros peces reales.

Resulta que el movimiento del cuerpo de la danza actúa como un superestímulo. Normalmente el vocabulario de una coreografía se divide en unidades que son gestos o movimientos simples. Estos son amplificados a través de un estilismo grácil, su cadencia, trayectoria o velocidad, lo que se puede denominar como fluidez perceptual, convirtiéndolos en fácilmente percibidos como movimiento biológico y procesados por nuestros sistemas de observación, entre ellos las NE. A su vez, sirven de input o entrada para alimentar nuestra maquinaria cognitiva, entre otras, de la cognición social (habilidad sociocognitiva para entender a otros) que finalmente nos permite entender la intencionalidad y el porqué de lo que vemos. Pero el poder simbólico de las acciones y su interpretación no es la única dimensión en esta nueva concepción de la filosofía de la danza. Esta es la segunda tesis que permite construir una nueva filosofía de la danza:

- Los movimientos del cuerpo en el arte de la danza que tienen expresión simbólica gracias a la percepción visual de los mismos como movimiento biológico adquieren también una apreciación estética.

Esta apreciación o valoración estética es mayor cuanto más difícil o imposible de realizar para el observador es una acción o movimiento corporal: el espectador sabe que está viendo una acción física que supera sus propias habilidades y esto produce un mayor agrado estético (Cross E., Kirsch L., Ticini L. y Schütz-Bosbach S. 2014). 
Con todos estos datos se crean dos tesis que hacen posible una nueva concepción de la filosofía de la danza. Estas dos tesis definen la danza como un arte representativo que produce efectos estéticos. La danza es representativa porque nuestra cognición visual (mecanismos perceptivos) desambigua las acciones del cuerpo como movimiento biológico, un fenómeno por el cual nuestra visión obtiene información relevante del cuerpo interpretándolo como intencional y, por tanto, otorgándole expresividad simbólica. Paralelamente, el sistema de observación de las acciones y en concreto las NE permiten la simulación en la mente del observador de las acciones y entenderlas como si las hiciera él. En la medida en que la ejecución física de los movimientos resulta difícil ejecutarlas simulacionalmente, porque supera las habilidades físicas del observador, el agrado o valoración estética es mucho mayor.

Los puntos clave de esta nueva concepción de la filosofía de la danza desde un enfoque naturalista son varios. Uno de ellos es que los movimientos estilizados del cuerpo en su expresión artística en la danza producen un efecto en nuestras capacidades mentales que en última instancia dan lugar a una respuesta estética. Otro es que los patrones de movimiento que vemos en la coreografía de una danza son un reflejo especular de las estructuras subyacentes de nuestra mente/cerebro.

La danza es una forma de arte representativo y simbólico como hemos ido viendo a lo largo de este escrito gracias a la evolución de una arquitectura cognitiva y sensorial dedicada a atender a los gestos del cuerpo y asignarles un significado (simbolismo). No obstante, la danza como forma de arte que utiliza el cuerpo como plataforma de expresión simbólica tiene límites. No todas las situaciones pueden ser representadas en la danza. Una representación o coreografía de danza es muy poco probable que cause emociones de disgusto, culpa, orgullo... principalmente porque las señales del movimiento biológico no inervan las partes del encéfalo asociadas con estas respuestas afectivas. La filosofía de la danza o la teoría estética y análisis crítico tiene que darse cuenta de lo que puede estar y no puede estar en una representación o coreografía de danza. Las ausencias son igual de importantes, a veces más que las presencias, sobre todo en el arte, pero esto puede dar pistas a la hora de las recensiones culturales del arte coreográfico.

También es importante señalar, porque este es otro punto clave de esta nueva concepción de la filosofía de la danza desde un enfoque naturalista, las implicaciones terapéuticas. La sincronización de los patrones de movimiento corporal con nuestro compañero/a de baile aumenta el vínculo y apego con aquél o aquella. Pero es que además la sincronización durante el baile alivia el dolor. Un reciente estudio (Tarr B., Launay J., Cohen E y Dunbar R. 2015) muestra cómo aquellos que bailan sincronizados con otros son capaces de soportar 20 unidades de presión más, indicando que su sensibilidad al dolor se ha reducido debido a liberación de péptidos endógenos como las endorfinas que se produce durante la sincronización del baile. Este estudio se suma a otros que han señalado como el fenómeno de sincronizarte con alguien (bailando, siguiendo el ritmo, andando etc.) aumenta la confianza, la empatía y genera cohesión grupal.

La compleja coordinación que requiere la danza, no ya con tu pareja de baile, sino en el propio individuo a nivel sensoriomotor, apunta a los enormes efectos beneficiosos. Efectos beneficiosos claros en términos neurológicos porque bailar es un "doble placer" que estimula la plasticidad cerebral y previene de procesos neurodegenerativos. Es un "doble placer" por- 
que la música activa los centros de recompensa del cerebro (o encéfalo) y al mismo tiempo el movimiento activa los circuitos sensoriales y motores. Este "doble placer", finalmente, estimula y favorece la salud del cerebro (Verghese et al. 2003).

Ver la filosofía de la danza desde el enfoque naturalista de las ciencias cognitivas donde el cuerpo tiene capacidad de expresión simbólica nos permite entender y explicar mejor uno de los universales humanos más característicos, a saber, la danza; y a su vez, la danza nos permite explorar la variedad de instanciaciones del cuerpo y su expresión simbólica.

\section{Discusiones}

Hemos presentado, brevemente, evidencias de la ciencia cognitiva de la danza para construir una filosofía de la danza desde un enfoque naturalista que parta de la premisa de que la danza es un tipo de arte representativo y simbólico. Esta nueva definición para el estudio filosófico de la danza contrasta con la tradicional definición de la danza como arte no-representativo. Creemos que entre las virtudes de esta nueva y preliminar definición de la danza como objeto de análisis para una filosofía de la danza desde una perspectiva naturalista se encuentra la posibilidad de distinguir qué es y qué no es danza y cuáles son sus funciones, así como la creación de teorías que puedan establecer generalizaciones sobre el fenómeno de la danza.

\section{Bibliografía}

Bresnahan A. (2015), “The Philosophy of Dance" en E. Zalta (ed) The Stanford Encyclopedia of Philosophy. Publicado el 12 de enero del 2015. Disponible en: http://plato. stanford.edu/entries/dance/

Brownlow S., Dixon A., Egbert C. y Radcliffe R. (1997), "Perception of movement and dancer characteritics from point light displays of dance". Psychol. Rec. No. 47, pp. 411-421.

Calvo-Merino B., Glaser D., Grezes J., Passingham R. y Haggard P. (2005), “Action observation and acquired motor skills: An fMRI study with expert dancers". Cerebral Cortex, No. 15, pp. 1905-1910.

Carroll N, (2004), "Mass art as art: A response to John Fisher". Journal of Aesthetics and Art Criticism, No. 62, pp. 61-65.

Christensen J, Nadal M., Cela-Conde C. y Gomila T. (2014), “A norming study and library of 203 dance movements". Perception, No. 43, pp. 178-206.

Clark A. y Chalmers D. (1998), “The extended mind”. Analysis, No. 58, pp. 7-19.

Cross E., Kirsch L., Ticini L. y Schütz-Bosbach S. (2014), "The impact of aesthetic evaluation and physical ability on dance perception", Front. Hum. Neurosci. No 8, pp. 102.

Fadiga L. et al. (1995), "Motor facilitation during action observation: a magnetic stimulation study". J. Neurophysiol, No. 73, pp. 2608-2611.

Freedberg D. y Gallese V. (2007), "Motion, emotion and empathy in esthetic experience". Trends Cogn. Sci., No. 8, pp. 396-403.

Gallese V., Fadiga L., Fogassi L. y Rizzolatti G. (1996), “Action recognition in the premotor cortex". Brain, No. 119, pp. 593-609. 
Gallese V. y Cuccio V. (2015), “The paradigmatic body: Embodied simulation, intersubjetivity and the bodily self”. En T. Metzinger y J. Windt (eds), Open Mind, 1-27. Frankfurt: MIND Group.

Hagendoorn I. (2011), "Dance, choreography and the brain” en D. Melcher y F. Bacci (eds) Art and the Senses. Oxford: Oxford University Press.

Johansson G. (1973), "Visual perception of biological motion and a model for its analysis". Perception and Psychophysics. N. 14, pp. 201-211.

Kandel E. (2016), Reductionism in Art and Brain Science: Bridging the Two Cultures. New York: Columbia University Press.

Kozlowski L. y Cutting J. (1977), "Recognizing the sex of a walker from a dynamic pointlight display". Perception and Psychophysics. No. 21, pp. 575-580.

Meltzoff A. (2013), "Origins of social cognition: Bidirectional self-other mapping and the like-me hypothesis" en M. Banaji y S. Gelman (eds), Navigating the Social World: What Infants, Children and Other Species Can Teach Us. New York: Oxford University Press.

Rizzolatti G., Fadiga L. Gallesse V. y Fogassi L. (1996a), "Premotor cortex and the recognition of actions". Cogn. Brain Res, No. 3, pp. 131-141.

Rizzolatti G. and Craighero L. (2004), "The Mirror Neuron System”. Annual Rev. Neurosci, No. 27, pp. 169.192

Tarr B., Launay J., Cohen E y Dunbar R. (2015), "Synchrony and exertion during dance independently raise pain threshold and encourage social bonding". Biology Letters, No. 11, pp. 20150767.

Verghese J. et al (2003), "Leisure Activities and the Risk of Dementia in the Elderly". $N$. Engl. J. Med. No. 348, pp. 2508-2516.

Zeki S. (2009), Splendors and Miseries of the Brain: Love, Creativity, and the Quest for Human Happiness. Sussex: Wiley-Blackwell. 
